\title{
ARTIGO 12
}

\section{QUALIDADE DE VIDA DE PACIENTES COM DOENÇA RENAL CRÔNICA}

Raquel de Sousa Sales Santos ${ }^{1}$, Ana Hélia de Lima Sardinha ${ }^{1}$

Objetivo: avaliar a Qualidade de Vida dos pacientes com Doença Renal Crônica Terminal em tratamento hemodialítico. Metodologia: estudo descritivo quantitativo, com questionários para os aspectos sociodemográficos e de saúde e o Kidney Disease and Quality of Life Short-Form. Resultados: pacientes na faixa etária 31-50 anos, sexo feminino, pardos, casados, 1-4 filhos, ensino fundamental incompleto, 06-10 anos de hemodiálise e comorbidades como hipertensão, cardiopatia e diabetes. Menores escores nas dimensões: papel profissional, função física, sobrecarga da doença renal e saúde geral. Discussão: A qualidade de vida dos pacientes sofreu alterações. Conclusão: Identificou-se aspectos que requerem atenção da equipe multiprofis-sional de saúde.

Descritores: Qualidade de Vida, Insuficiência Renal Crônica, Diálise Renal.

\section{QUALITY OF LIFE OF PATIENTS WITH CHRONIC RENAL DISEASE ABSTRACT}

Objective: to evaluate the Quality of Life of patients with Terminal Chronic Kidney Disease under hemodialysis treatment Methodology: quantitative descriptive study, with questionnaires for sociodemographic and health aspects and Kidney Disease and Quality of Life Short-Form. Results: patients aged 31-50 years, female, brown, married, 1-4 children, incomplete elementary school, 6-10 years of hemodialysis and comorbidities: hypertension, cardiopathy and diabetes. Lower scores on the dimensions: professional role, physical function, renal disease overload and general health. Discussion: Patients' quality of life has changed. Conclusion: It was identified aspects that require greater attention from the multiprofessional health team.

Descriptors: Quality of Life, Renal Insufficiency Chronic, Renal Dialysis.

\section{CALIDAD DE VIDA DE LOS PACIENTES CON ENFERMEDAD RENAL CRÓNICA RESUMEN}

Objetivo: evaluar la calidad de vida de los pacientes con enfermedad renal crónica terminal en tratamiento de hemodiálisis. Metodología: estudio descriptivo cuantitativo con cuestionarios concernientes a los aspectos demográficos, de salud y el Kidney Disease and Quality of Life Short-Form. Deducciones: los pacientes de edad entre 31-50 años, género femenino, pardos, casados, tienen 1-4 hijos, la educación primaria incompleta, 06-10 años conviviendo de forma simultánea con la hemodiálisis y las enfermedades como hipertensión, problemas cardiacos y diabetes. Las valoraciones más bajas en las dimensiones: función profesional, la función física, la sobrecarga de la enfermedad renaly la salud general. Discusión: la calidad de vida de los pacientes ha cambiado. Conclusión: se ha identificado los aspectos que demandan la atención del equipo de salud multidisciplinario. Descriptores: Calidad de Vida, Insuficiencia Renal Crónica, Diálisis Renal. 


\section{INTRODUÇÃO}

A Doença Renal Crônica (DRC) é uma lesão do parênquima renal e/ou a diminuição da taxa de filtração glomerular presentes por um período igual ou superior a três meses. Sendo assim, uma síndrome complexa consequente à perda da capacidade excretória renal, geralmente lenta e progressiva ${ }^{1}$

A partir desta definição, propôs-se a classificação com cinco estagiamentos para a DRC. Neste estudo aborda-se o estágio $V$ ou terminal, que compreende a falência funcional renal, com filtração glomerular menor que $15 \mathrm{~mL} / \mathrm{min} / 1,73 \mathrm{~m} 2$, havendo a necessidade de início de terapia de substituição da função renal ${ }^{2}$.

À medida que a DRC Terminal (DRCT) avança, um conjunto de sinais e sintomas que constituem a síndrome urêmica podem aparecer, sendo eles: fadiga, emagrecimento, prurido, náuseas, insônia, comprometimento do estado mental e sintomas neuromusculares como espasmos, fraqueza muscular e câimbras ${ }^{3}$.

As modalidades de tratamento da DRCT para substituição das funções renais são: a diálise, que se subdivide em diálise peritoneal e hemodiálise e o transplante renal. A modalidade mais utilizada é a hemodiálise, tipo de tratamento realizado por uma máquina para remover líquidos e produtos do metabolismo do corpo, com uma média de três sessões semanais e período de três a cinco horas/sessão, dependendo das necessidades individuais do paciente ${ }^{4}$.

Segundo o último censo da Sociedade Brasileira de Nefrologia, o total de pacientes em tratamento dialítico é de 112.004, sendo os tipos de diálise por fonte pagadora: Sistema Único de Saúde (SUS) - hemodiálise (91,3\%) e diálise peritoneal (5,2\%); não SUS - hemodiálise $(85,0 \%)$ e diálise peritoneal $(7,8 \%)^{5}$

A DRCT e a hemodiálise estão entre as patologias e as terapias de caráter crônico que mais afetam negativamente a Qualidade de Vida ( $\mathrm{QV}$ ) dos pacientes. Tal fato decorre de fatores como o convivio com uma doença incurável, considerada grande problema de saúde pública por suas elevadas taxas de morbidade e mortalidade; dependência de uma máquina; esquema terapêutico rigoroso; alterações na imagem corporal e restrições dietéticas e hídricas ${ }^{6}$.

A Organização Mundial de Saúde define a OV como "a percepção que o indivíduo possui de sua vida e que envolve um sistema de cultura e valores aos quais ele vive, relacionandoos com seus objetivos, expectativas, padrões e preocupações". Reiterando que a OV referente à saúde, remete ao modo como a patologia e o tratamento influenciam a percepção das pessoas envolvidas no contexto de saúde-doença7.

Dessa forma, a OV tem se tornado importante critério na avaliação da efetividade de tratamentos e intervenções na área da saúde. É necessário avaliar indicadores de funcionamento físico, aspectos sociais, estado emocional e mental, da repercussão de sintomas e da percepção individual de bem-estar ${ }^{6}$

Portanto, durante a assistência aos pacientes com DRCT em hemodiálise, surgiu o interesse em compreender o impacto dessa doença e seu tratamento na OV desses pacientes, analisando o contexto e considerando as repercussões em seus cotidianos.

Há estudos ${ }^{8-19}$ nessa temática desenvolvidos por diferentes profissionais, utilizado o Kidney Disease and Quality-of-Life Short-Form (KDOOL-SFTM 1.3), importante instrumento que avalia a OV em pacientes com DRCT, traduzido para o idioma português, além de outros como espanhol, alemão, francês e japonês ${ }^{20}$

Contudo, a avaliação dos aspectos da OV requer constante atualização, devido às possibilidades de mudanças pela temporalidade e na individualidade das pessoas. Alterações na OV são bases para formulações de estratégias de intervenção, resolução de problemas específicos e suporte especializado visando minimizar os efeitos da doença de caráter progressivo. Diante disso, este estudo objetivou avaliar a Qualidade de Vida dos pacientes com Doença Renal Crônica Terminal em tratamento hemodialítico.

\section{METODOLOGIA}

Estudo descritivo com abordagem quantitativa, realizado na Unidade Renal do Hospital Universitário da Universidade Federal do Maranhão, no periodo de setembro a novembro de 2016.

A população foi constituída por pacientes que satisfizeram os critérios de inclusão: apresentar diagnóstico de DRCT, submetido à hemodiálise e idade igual ou superior a 18 anos. Foram excluídos pacientes com déficit intelectual (registrados no prontuário), dificuldades orgânicas (aspecto clínico, deficiência auditiva e neurodegenerativas), que impossibilitavam a aplicação dos questionários ou que estivessem internados.

O tamanho da amostra foi estimado em 52 pacientes, com erro amostral de $5 \%$ e nível de confiança de $95 \%$. Destes, 10 se recusaram a participar, 02 foram transplantados e 02 faleceram. Dessa forma, participaram deste estudo 38 pacientes.

Na coleta de dados foram utilizados dois questionários: o elaborado pelas pesquisadoras, que identificou os aspectos sociodemográficos e de saúde e o Kidney Disease and Qualityof-Life Short-Form (KDOOL-SFTM 1.3) traduzido no Brasil para "Sua Saúde e Bem-Estar - Doença Renal e Qualidade de Vida"20.

O KDOOL-SFTM é um instrumento específico para avaliar 
QV em pacientes com DRCT, constituído de 80 itens, divididos em 19 escalas, aplicável a pacientes que realizam algum tipo de diálise. Seus escores próximos à zero indicam pior estado de $\mathrm{OV}^{20}$.

Ambos os questionários foram lidos para os pacientes, que escolhiam a resposta que melhor se adequava ao seu caso, enquanto estavam submetidos à sessão de hemodiálise.

Os dados do questionário que identificou os aspectos sociodemográficos e de saúde foram avaliados utilizando estatística descritiva, trabalhando com a frequência dos eventos no programa Epi Info ${ }^{T M}$ 7, desenvolvido pelo Centers for Disease Control and Prevention.

Os valores referentes ao instrumento KDOOL-SFTM foram agregados e descritos no software KDOOL-SF ${ }^{\top M} 1.3$ Scoring Program (v2.0), disponibilizado pelo site do KDOOL Working Group, específico para este questionário.

Foi obtido aprovação do Comitê de Ética em Pesquisa do Hospital Universitário da Universidade Federal do Maranhão após análise, segundo a Resolução nํ466/12 do Conselho Nacional de Saúde, com parecer ${ }^{\circ} 1.700 .736$. Os participantes assinaram o Termo de Consentimento Livre e Esclarecido.

\section{RESULTADOS}

Entre os 38 pacientes participantes, evidenciou-se nos aspectos sociodemográficos: 50,00\% na faixa etária de 3150 anos, 55,26\% do sexo feminino, 73,68\% da cor/raça parda, $68,42 \%$ casados, $60,53 \%$ que possuíam 1 a 4 filhos e $39,47 \%$ que apresentavam como nível de escolaridade o ensino fundamental incompleto (Tabela 1).

Tabela 1: Aspectos sociodemográficos dos pacientes com doença renal crônica terminal, em tratamento hemodialítico, São Luís-MA, 2016.

\begin{tabular}{|c|c|c|}
\hline Variáveis & Frequência (n=38) & Porcentagem $(100 \%)$ \\
\hline \multicolumn{3}{|l|}{ Idade (anos) } \\
\hline $18-30$ & 6 & $15,79 \%$ \\
\hline $31-40$ & 11 & $28,95 \%$ \\
\hline $41-50$ & 8 & $21,05 \%$ \\
\hline $51-60$ & 7 & $18,42 \%$ \\
\hline $61-70$ & 4 & $10,53 \%$ \\
\hline $71-80$ & 2 & $5,26 \%$ \\
\hline \multicolumn{3}{|l|}{ Sexo } \\
\hline Feminino & 21 & $55,26 \%$ \\
\hline Masculino & 17 & $44,74 \%$ \\
\hline \multicolumn{3}{|l|}{ Cor/raça } \\
\hline Parda & 28 & $73,68 \%$ \\
\hline Preta & 10 & $26,32 \%$ \\
\hline \multicolumn{3}{|l|}{ Estado civil } \\
\hline Casado & 26 & $68,42 \%$ \\
\hline Solteiro & 8 & $21,05 \%$ \\
\hline Separado & 3 & $7,89 \%$ \\
\hline Divorciado & 1 & $2,63 \%$ \\
\hline \multicolumn{3}{|l|}{ Filhos } \\
\hline Não & 6 & $15,79 \%$ \\
\hline $1-2$ & 12 & $31,58 \%$ \\
\hline $3-4$ & 11 & $28,95 \%$ \\
\hline 5 ou mais & 9 & $23,68 \%$ \\
\hline \multicolumn{3}{|l|}{ Nível de escolaridade } \\
\hline Não alfabetizado & 4 & $10,53 \%$ \\
\hline Alfabetizado & 2 & $5,26 \%$ \\
\hline Ens. fundamental completo & 4 & $10,53 \%$ \\
\hline $\begin{array}{l}\text { Ens. fundamental } \\
\text { incompleto }\end{array}$ & 15 & $39,47 \%$ \\
\hline Ens. médio completo & 10 & $26,32 \%$ \\
\hline Ens. médio incompleto & 3 & $7,89 \%$ \\
\hline
\end{tabular}

Em relaçãoaosaspectos de saúde, notempo detratamento hemodialítico $36,84 \%$ dos pacientes encontravam-se no intervalo 06-10 anos, seguido por 31,58\% no intervalo 02-05 anos. Acerca das comorbidades que mais acometeram os pacientes, constatou-se: hipertensão (51,85\%), cardiopatia $(12,96 \%)$ e diabetes (11,11\%), havendo pacientes que apresentaram mais de uma comorbidade (Tabela 2).

Tabela 2 - Aspectos de saúde dos pacientes com doença renal crônica terminal, em tratamento hemodialítico, São Luís-MA 2016.

\begin{tabular}{|c|c|c|}
\hline Variáveis & Frequência $(n=38)$ & Porcentagem $(100 \%)$ \\
\hline \multicolumn{3}{|c|}{$\begin{array}{l}\text { Tempo de hemodiálise } \\
\text { (anos) }\end{array}$} \\
\hline $02-05$ & 12 & $31,58 \%$ \\
\hline $06-10$ & 14 & $36,84 \%$ \\
\hline $11-15$ & 8 & $21,05 \%$ \\
\hline $16-18$ & 4 & $10,52 \%$ \\
\hline \multicolumn{3}{|l|}{ Comorbidades* } \\
\hline Nenhuma & 7 & $12,96 \%$ \\
\hline Hipertensão & 28 & $51,85 \%$ \\
\hline Diabetes & 6 & $11,11 \%$ \\
\hline Cardiopatia & 7 & $12,96 \%$ \\
\hline Outras diversas & 6 & $11,11 \%$ \\
\hline
\end{tabular}

No referente aos escores das dimensões da OV do KDQOLSFTM com valores médios baixos, portanto componentes prejudicados da OV, temos: Papel profissional $(21,05)$. Função física $(25,66)$, Sobrecarga da doença renal $(35,86)$ e Saúde geral $(43,55)$ (Tabela 3).

Tabela 3 - Dimensões da qualidade de vida $\left(\mathrm{KDOOL}^{-S F^{\mathrm{TM}}}\right)$ dos pacientes com doença renal crônica terminal, em tratamento hemodialítico, São Luís-MA, 2016 (n=38).

\begin{tabular}{lccc}
\hline Dimensões - (número de itens na escala) & Média & Mediana & Desvio Padrão \\
\hline Sintomas/problemas (12) & 80,10 & 83,33 & 14,16 \\
Efeitos da doença renal (8) & 71,22 & 71,88 & 14,68 \\
Sobrecarga da doença renal (4) & 35,86 & 31,25 & 25,07 \\
Papel profissional (2) & 21,05 & 0,00 & 29,94 \\
Função cognitiva (3) & 85,26 & 86,67 & 14,47 \\
Qualidade de interação social (3) & 81,58 & 86,67 & 17,01 \\
Função sexual (2) & 92,71 & 100,00 & 13,55 \\
Sono (4) & 74,93 & 77,50 & 18,77 \\
Suporte social (2) & 78,51 & 83,33 & 21,54 \\
Estimulo da equipe de diálise (2) & 75,33 & 87,50 & 26,71 \\
Satisfação do paciente (1) & 54,39 & 50,00 & 16,30 \\
Funcionamento físico (10) & 57,76 & 70,00 & 33,99 \\
Função física (4) & 25,66 & 0,00 & 36,98 \\
Dor (2) & 68,36 & 70,00 & 28,05 \\
Saúde geral (5) & 43,55 & 45,00 & 20,73 \\
Bem estar emocional (5) & 69,37 & 72,00 & 19,84 \\
Função emocional (3) & 54,39 & 66,67 & 44,80 \\
Função social (2) & 75,00 & 75,00 & 22,51 \\
Energia/Fadiga (4) & 62,37 & 60,00 & 21,24 \\
\hline
\end{tabular}

\section{DISCUSSÃO}

Considerando os aspectos sociodemográficos, percebeuse que os pacientes com DRCT em tratamento hemodialitico estão na fase adulta, sendo maioria do sexo feminino, pardos, casados, com filhos e baixo nivel de escolaridade.

Em relação à idade, foram encontrados estudos com uma média de 51 e 56 anos $^{8,10}$ e faixas etárias de 40-60 anos $^{11,12}$ ou $50-59$ anos $^{13,14}$ e alguns $s^{8,14}$ mostraram que quanto 
maior a idade, maiores as dificuldades pessoais enfrentadas, resultando em menores escores nas dimensões da OV.

A respeito do sexo, há na literatura tanto feminino ${ }^{10.15}$ quanto masculino ${ }^{16,17}$, ressaltando que quando é feito comparações nestes estudos as mulheres obtém índice geral menor de $\mathrm{OV}$, existindo diferenças na maneira de se adaptar e enfrentar a doença. Estas devem ser levadas em consideração no processo terapêutico.

Acerca do estado civil, evidenciou-se predomínio dos casados $^{10.16,17}$, havendo identificações como pacientes com companheiro fixo ${ }^{14}$, em estudos que demonstraram que este fato pode contribuir de forma positiva no cuidado em domicílio e na realização de atividades habituais pelos pacientes com autonomia comprometida.

No nível de escolaridade identificou-se uma pesquisa em que apenas $38,9 \%$ dos pacientes sabiam ler e escrever ${ }^{8}$ e outra na qual $63,2 \%$ possuíam baixa escolaridade ${ }^{14,18}$, corroborando com o encontrado neste estudo. Foi descrito por autores ${ }^{11,14}$ que a escolaridade menor que oito anos apresenta associação com niveis baixos de OV.

Quanto aos aspectos de saúde, no tempo de tratamento hemodialítico a maioria dos pacientes encontravam-se no intervalo 06-10 anos (72-120 meses). Pesquisas mostraram um tempo médio de 43,15 meses ${ }^{16}$ e 77,5 meses ${ }^{14}$, expondo que o maior tempo de hemodiálise influencia negativamente os componentes da OV.

As comorbidades referidas pelos pacientes nesta pesquisa estão entre as consideradas nos grupos de risco para DRC ${ }^{2}$, ou seja, nos quais os pacientes apresentam suscetibilidade aumentada, sendo eles: hipertensos, diabéticos e pacientes com doença cardiovascular, além dos idosos, familiares de pacientes com DRC e pacientes em uso de medicações nefrotóxicas.

O convívio com uma DRC reflete modificações tanto na vida do paciente como na de seus familiares, pois as limitações impostas pelo tratamento ocasionam diversas alterações, que interferem na sua qualidade de vida, influenciando na manutenção do tratamento hemodialitico e propiciando ao paciente dificuldades de aspecto social ${ }^{21}$.

Analisando os escores das dimensões da OV do KDQOLSFTM que apresentaram menores escores, percebe-se que a DRCT e o tratamento hemodialítico repercutem de forma prejudicial na vida dos pacientes, comprometendo alguns fatores da QV.

As dimensões Papel profissional e Função física foram encontradas entre as mais prejudicadas em pesquisas semelhantes ${ }^{10,16,17}$ realizadas nas diversas regiões do país, permanecendo entre as menores no nosso estado ${ }^{11,13}$.

$\mathrm{Na}$ dimensão Papel profissional, que avalia se os pacientes acreditam que a doença os impediu de exercer trabalho remunerado, identificou-se que a DRCT representa empecilho para que os pacientes tenham oportunidade de adquirir trabalho e dificuldade para que se mantenham empregados e podendo colaborar com a renda familiar, pois precisam se afastar do trabalho para frequentar as sessões de hemodiálise ${ }^{9.1718}$, com comparecimento obrigatório.

A Função física, referente ao tipo e quantidade de trabalho ou atividades habituais e a dificuldade em realizá-los, contribuiu para diminuição na OV por conta das repercussões musculoesqueléticas, comuns em pacientes submetidos à hemodiálise, podendo estes apresentarem diminuição do desempenho funcional e prejuízo na prática de atividade física ${ }^{15}$.

A Sobrecarga da doença renal, representada pela interferência da DRCT na vida do paciente, a decepção em ter que lidar com a doença e o sentimento de peso para a família, foi descrita em estudos ${ }^{11,12,17}$ como fator que altera a OV.

A baixa média na dimensão Saúde geral expressa que os pacientes compreendem que ficam doentes com mais facilidade que outras pessoas, não se sentem saudáveis e acreditam que sua saúde pode piorar, assim como descrevem outros pesquisadores ${ }^{16,19}$

Alguns autores que também utilizaram o instrumento KDQOL-SFTM encontraram outras dimensões com menores escores: Função sexual9,19, Função emocional ${ }^{8,14}$, Satisfação do paciente ${ }^{11}$ e Funcionamento físico ${ }^{16}$. Estes baixos escores permitem que sejam feitas inferências, direcionando a atenção dos profissionais para os aspectos que estão influenciando ou modificando a vida dos pacientes ${ }^{8}$.

Como limitações neste estudo, ressaltam-se as peculiaridades do tema pesquisado, focado em grupo especifico de pacientes, realizado durante sessões de hemodiálise e em um só hospital. Todavia, partimos da premissa que este representa um marco motivacional para o desenvolvimento e aprofundamento de novas pesquisas contidas na mesma linha investigativa em outras unidades de saúde. Destaca-se, ainda, que não houve conflito de interesses por parte das autoras.

\section{CONCLUSÃO}

Constatou-se que a qualidade de vida dos pacientes sofreu alterações, sendo afetada negativamente nas dimensões: papel profissional, função física, sobrecarga da doença renal e saúde geral, o que pode ser atribuído às limitações provocadas pela doença renal crônica terminal e seu tratamento.

Esta pesquisa identificou aspectos que requerem maior atenção da equipe multiprofissional de saúde, dando subsídio para a implementação de novas estratégias e melhora da sobrevida dos pacientes. A assistência profissional, além do apoio familiar e de amigos, favorece o enfrentamento da doença e seu tratamento, minimizando as frustrações impostas na rotina de vida dos pacientes. 


\section{REFERÊNCIAS}

1. Noleto LC, Fonsêca AC, Luz MHBA, Batista OMA, Pereira AFM. O papel dos profissionais de enfermagem no cuidado ao paciente em tratamento hemodialítico: revisão integrativa. Rev enferm UFPE [Internet]. 2015 [cited 2016 Set 10];9(10):1580-6.

2. Group KDIGO. Clinical Practice Guideline for the Evaluation and Management of Chronic Kidney Disease. Kidney International Supplements. Journal of the International Society of Nephrology [Internet]. 2013 [cited 2016 Out 15];3(1):1-150

3. Scatolin BE, Vechi AP, Ribeiro DF, Bertolin DC, Canova JCM, Cesarino CB, Ribeiro RCHM. Atividade de vida diária dos pacientes em tratamento de diálise peritoneal intermitente com cicladora. Arq Ciênc Saúde [Internet]. 2010 [cited 2016 Set 10];17(1):15-21.

4. Riella MC. Principios de nefrologia e distúrbios hidroeletrolíticos. 5.ed. Rio de Janeiro: Guanabara Koogan; 2010.

5. Sesso RC, Lopes AA, Thomé FS, Lugon JR, Martins CT. Inquérito Brasileiro de Diálise 2014. J Bras Nefrol [Internet]. 2016 [cited 2017 Jan 25];38(1):54-61.

6. Martins MRI, Cesarino CB. Qualidade de Vida de Pessoas com Doença Renal Crônica em Tratamento Hemodialítico. Rev Latino-am Enfermagem [Internet]. 2005 [cited 2016 Out 15];13(5):670-6.

7. The WHOQOL Group. The World Health Organization quality of life assessment (WHOQOL): position paper from the World Health Organization. Soc Sci Med [Internet]. 1995 [cited 2016 Nov 20]:41:140310 .

8. Cordeiro JABL, Brasil VV, Silva AMTC, Oliveira LMAC, Zatta LT, Silva ACCM. Qualidade de vida e tratamento hemodialítico: avaliação do portador de insuficiência renal crônica. Rev Eletr Enf [Internet]. 2009 [cited 2016 Nov 20];11(4):785-93.

9. Poersch RF, Andrade FP, Dal Bosco A, Rovedder PME. Qualidade de vida em pacientes com doença renal crônica submetidos à hemodiálise. ConScientia e Saúde [Internet]. 2015 [cited 2016 Dez 01];14(4):608-616.

10. Qualidade de vida de pacientes com insuficiência renal em tratamento hemodialítico. Rev Enferm UFPE [Internet]. 2017 [cited 2018 Jan 30];11(11):4663-70.

11. Cavalcante MCV, Lamy ZC, Lamy Filho F, França AKTC, Santos AM, Thomaz EBAF, Silva AAM, Salgado Filho N. Fatores associados à qualidade de vida de adultos em hemodiálise em uma cidade do nordeste do Brasil. J Bras Nefrol [Internet]. 2013 [cited 2016 Dez $01] ; 35(2): 79-86$
12. Grasselli CSM, Chaves ECL, Simão TP, Botelho PB, Silva RR. Avaliação da qualidade de vida dos pacientes submetidos à hemodiálise. Rev Bras Clin Med [Internet]. 2012 [cited 2016 Out 15];10(6):503-7.

13. Coutinho NPS, Vasconcelos GM, Lopes MLH, Wadie WCA, Tavares $\mathrm{MCH}$. Qualidade de vida de pacientes renais crônicos em hemodiálise. Rev Pesq Saúde [Internet]. 2010 [cited 2016 Nov 20];11(1):13-17.

14. Silva AC, Coelho DM, Diniz GCLM. Qualidade de vida dos pacientes com insuficiência renal crônica submetidos à hemodiálise em um hospital público de Betim, Minas Gerais. Sinapse Múltipla [Internet]. 2012 [cited 2016 Set 10]:1(2):103-13.

15. Costa PB, Vasconcelos KFS, Tassitano RM. Qualidade de vida: pacientes com insuficiência renal crônica no municipio de Caruaru, PE. Fisioter Mov [Internet]. 2010 [cited 2016 Dez 01];23(3):461-71.

16. Lopes JM, Fukushima RL, Inouye K, Pavarini SC, Orlandi FS. Qualidade de vida relacionada à saúde de pacientes renais crônicos em diálise. Acta Paul Enferm [Internet]. 2014 [cited 2016 Nov 20];27(3);2306.

17. Silva GD, Fernandes BD, Silva FA, Dias YCB, Melchiors AC, Qualidade de vida de pacientes com insuficiência renal crônica em tratamento hemodialítico: análise de fatores associados. R. bras Qual Vida [Internet]. 2016 [cited 2017 Jan 25];8(3):229-245.

18. Marinho CLA, Oliveira JF, Borges JES, Silva RS, Fernandes FECV. Qualidade de vida de pessoas com doença renal crônica em hemodiálise. Rev Rene [Internet]. 2017 [cited 2018 Jan 30];18(3):396403.

19. Zanesco C, Giachini E, Abrahão CAF, Silva DTR. Qualidade de vida em pacientes hemodialíticos: avaliação através do questionário KDQOLSF ${ }^{\mathrm{TM}}$. Rev Saúde Com [Internet]. 2017 [cited 2018 Jan 30]; 13(1):818-823.

20. Duarte OS, Miyazaki MCOS, Ciconelli RM, Sesso R. Tradução e Adaptação Cultural do Instrumento de Avaliação de Qualidade de Vida para pacientes Renais Crônicos (KDOOL-SFTM). Rev Assoc Med Bras [Internet]. 2003 [cited 2016 Dez 01]:49(4):37581. Disponi em: http://www.scielo.br/scielo.php?pid=SO10442302003000400027धscript=sci_abstractEtlng=pt

21. Ribeiro IP, Pinheiro ALS, Soares ALA, Santos NFM. Perfil Epidemiológico dos portadores de insuficiência renal crônica submetidos à terapia hemodialítica. Enferm. Foco [Internet].2014 [cited 2018 Jul 30];5(3/4):65-69. Disponivel em: http://revista.cofen. gov.br/index.php/enfermagem/article/viewFile/567/249 